\title{
Reliable Propagation of Magnetic Domain Walls in Cross Structures for Advanced Multiturn Sensors
}

\author{
B. Borie, ${ }^{1,2}$ M. Voto, ${ }^{3}$ L. Lopez-Diaz, ${ }^{3}$ H. Grimm, ${ }^{2}$ M. Diegel, ${ }^{4}$ M. Kläui, ${ }^{1, *}$ and R. Mattheis ${ }^{4, \dagger}$ \\ ${ }^{1}$ Institut für Physik, Johannes Gutenberg-Universität Mainz, Staudinger Weg 7, 55128 Mainz, Germany \\ ${ }^{2}$ Sensitec GmbH, Hechtsheimer Straße 2, Mainz D-55131, Germany \\ ${ }^{3}$ Departamento de Física Aplicada, Universidad de Salamanca, \\ Plaza de la Merced s/n, E-37001 Salamanca, Spain \\ ${ }^{4}$ Leibniz-Institut für Photonische Technologien, Albert-Einstein-Straße 9, 07745 Jena, Germany \\ (Received 13 June 2017; revised manuscript received 14 August 2017; published 13 October 2017)
}

\begin{abstract}
We develop and analyze an advanced concept for a domain-wall-based sensing of rotations. Moving domain walls in $n$ closed loops with $n-1$ intersecting convolutions by rotating fields, we are able to sense $n$ rotations. By combining loops with coprime numbers of rotations, we create a sensor system allowing for the total counting of millions of turns of a rotating applied magnetic field. We analyze the operation of the sensor and identify the intersecting cross structures as the critical component for reliable operation. Specifically, depending on the orientation of the applied field angle with the magnetization in the branches of the cross, a domain wall is found to propagate in an unwanted direction, yielding failures and counting errors in the device. To overcome this limiting factor, we introduce a specially designed syphon structure to the controlled pinning of the domain wall before the cross and depinning and propagation only for a selected range of applied field angles. By adjusting the syphon and the cross geometry, we find that the optimized combination of both structures prevents failures in the full sensor structure yielding robust operation. Our modeling results show that the optimized element geometry allows for the realization of the sensor with cross-shaped intersections and an operation that is tolerant to inaccuracies of the fabrication.
\end{abstract}

DOI: 10.1103/PhysRevApplied.8.044004

\section{INTRODUCTION}

Magnetic domain walls in soft ferromagnetic conduits have been under research since the mid-1960s [1-14]. Proposals using domain walls (DWs) in soft magnetic nanostructures [3,4] as key elements have been put forward to realize magnetic logic gates [5-8], memory devices such as racetrack memory, magnetic-bead transportation [10], and multiturn counter sensors with storage capability [11-14]. The first industrial-device realization was a multiturn counter, which can sense and store the number of turns with a true-power-on functionality. This sensor can count from 0 to 16 and back, it is manufactured by Novotechnik, and it is already commercially available (RSM-2800 Multiturn and RMB-3600 Multiturn [15]). The concept of this kind of sensor is the generation and movement of $180^{\circ} \mathrm{DWs}$ along giant magnetoresistance nanowires in an open-loop spiral-like geometry [see Fig. 1(a)]. Many industrial applications require a significant number of counts (from hundreds up to millions). However, the scaling of this particular geometry remains limited, and this number of counts can thus not be achieved.

A different approach was recently proposed based on using closed loops with a different number of cusps

\footnotetext{
*klaeui@uni-mainz.de

†roland.mattheis@leibniz-ipht.de
}

directed toward the center of the loop [14]. However, an issue inherent to the cusp geometry is the double width caused by the merging of two nanowires at this position. This characteristic imposes a reduction of the maximum sensed field before unwanted and uncontrolled random nucleation is initiated in this wider part. The cusp geometry thus narrows the field operating window (FOW) of the sensor, which is defined by the difference between the maximum magnetic field above which unwanted DW nucleation occurs and the minimum field necessary for a (a) Three-turn open-loop spiral

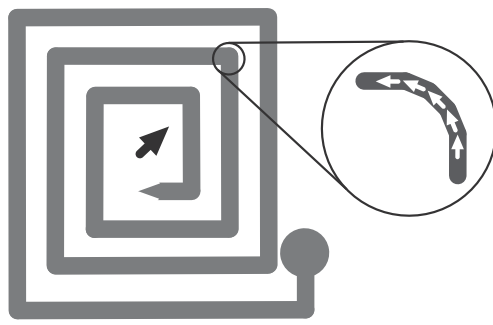

(b) Three-turn closed-loop

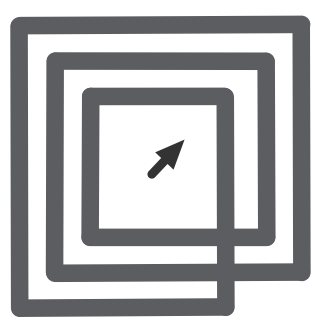

FIG. 1. (a) A three-turn open-loop spiral with a DW nucleation pad and a pointed end. The enlargement shows the polygonshaped corner with $22.5^{\circ}$ kink angles typically used [15]. (b) A three-turn closed-loop structure with two cross-shaped intersections. Note that, in a real device, the distance between two crosses is expected to be much larger than the wire width effectively decoupling the crosses. 
reliable transport of the DW throughout the whole structure. Compared to a perfectly straight stripe, the minimum field necessary for propagation through a cusp is also significantly increased. Thus, the FOW is narrowed by an increased minimum and a reduced maximum operating field, making this device geometry challenging for real use.

Because of the limitations imposed by the reduced FOW, there is a need for the development of alternative concepts. A possible different concept uses the combination of intersecting closed loops capable of counting coprime numbers of turns. This concept includes a different geometrical feature, namely, a cross of nanowires to allow the intertwining of loops [Fig. 1(b)] [16]. This geometry fundamentally allows for a much improved multiturn counter. We name such a structure an $n$-CL, where $n$ is the number of closed loops. For example, Fig. 1(b) shows a 3-CL device with three closed loops and two crossings that can count three turns. Every $n$-CL contains a minimum of 2 and a maximum of $2 n-2$ DWs for sensing.

An intrinsic characteristic of the closed-loop structure [Fig. 1(b)]—and, arguably, the essential one-is the possibility of automatically resetting back to counting from 0 after achieving the maximum number of turns offered by the architecture. Using this resetting mechanism with the concept of coprime number counting permits us to achieve counting of a much larger number of turns. This type of counting is allowed by positioning several $n$-CLs next to each other, with $n=3,5,7$, and so forth, with $n$ being the coprime number. The results of individual structures are combined to enable counting up to large numbers. The simultaneous use of a 3-CL, a 5-CL, and a 7-CL (Table I) yields different sequences of output states allowing a maximum count of $3 \times 5 \times 7=105$ turns, which is already more than any open-loop structure [Fig. 1(a)] could ever do. The method is scalable and, with seven different $n$-CLs ( $n=5,7,9,13,16,17$, and 19), the number of turns available is already $5 \times 7 \times 9 \times 13 \times 16 \times 17 \times 19 \approx\left(21 \times 10^{6}\right)$.

This closed-loop multiturn sensor thus opens up additional fields of applications where the open multiturn counter would be inefficient, such as, for example, for highly sensitive angle detection via pole wheels.

TABLE I. Table summarizing the output of the devices for different count numbers.

\begin{tabular}{lccc}
\hline \hline Maximum number of turns & 3-CL & 5-CL & 7-CL \\
\hline State of the device after 1 turn & 1 & 1 & 1 \\
2 turns & 2 & 2 & 2 \\
3 turns & 3 & 3 & 3 \\
4 turns & 1 & 4 & 4 \\
5 turns & 2 & 5 & 5 \\
6 turns & 3 & 1 & 6 \\
16 turns & 1 & 1 & 2 \\
31 turns & 1 & 1 & 3 \\
91 turns & 1 & 1 & 7 \\
106 turns & 1 & 1 & 1 \\
\hline \hline
\end{tabular}

However, a fundamental problem for this sensor operation arises at the eventuality that a DW does not propagate straight through the cross but instead takes a turn and thereby changes its path leading to a counting failure event. This event is particularly likely if the external field direction that drives the DW through the cross is not well controlled. Therefore, more sophisticated device geometries than the one shown in Fig. 1(b) are needed for reliable device operation, robustness, and fault tolerance.

In this paper, we present an alternative sensor architecture that reliably counts large numbers of complete turns of a rotating applied magnetic field. The structure is designed to comprise an alternative syphon-shaped element in addition to the cross-shaped intersections of nanowires [17]. This design allows for the desired control of the propagation direction of the DW under the application of a rotating field. First, we introduce the various possible states that can be present in the cross structure and explain the necessity for a syphon structure. Second, the angular dependence of the crossing and the syphon are separately simulated to extract the critical points of the two geometries. Finally, the results are combined to observe the behavior of a complete structure. We identify the three key parameters that allow us to gauge the reliability of the structure, and optimized devices are demonstrated for further improvements of the concept.

\section{CONCEPT FOR A DW-BASED MULTITURN COUNTER WITH CROSSES AND SYPHON ELEMENTS}

To overcome the limitations caused by the cusp geometry, we propose an alternative concept, schematically shown in Fig. 1(b). Here, the inner and outer ends of the spiral of Fig. 1(a) are connected via a nanowire, generating a cross-shaped intersection. In magnetically soft wires, the nucleation field for a DW follows a simple StonerWohlfarth-like model [18], and it is thus an inverse function of the stripe width. A cross leads to only a $\sqrt{2}$ fold increase of the width of the nanowire in its diagonal, compared to a twofold increase with the cusp geometry [14]. Quantitatively, with a 30-nm-thick and 300-nm-wide stripe of $\mathrm{Ni}_{81} \mathrm{Fe}_{19}$, the nucleation field is therefore improved by at least $40 \%$ by utilizing a cross structure.

The propagation of DWs in straight and curved wires is well established [4], and the motion of a DW through the cross has been studied $[19,20]$. However, the details of the reliability of this process are still under active investigation due to the complex dynamics involved in the process. In this section, which is dedicated to an explanation of the concept of the sensor mechanism, we use a transversedomain-wall type as a simple representation of a DW.

The quasistatic states before and after traveling through the cross are represented in Fig. 2. They illustrate the process of a moving DW in the horizontal arm from the left to the right side under conditions where the magnetization of the vertical arm is in an energetically favored state, 
Cross with straight arms and uncritical magnetization structure (a)

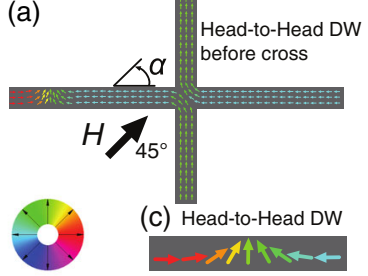

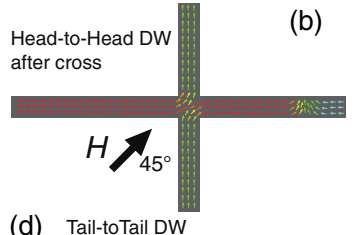

d) Tail-toTail DW
FIG. 2. Sketch of a DW configuration before and after moving across a cross with an energetically favored magnetization state of the vertically oriented arm. $\alpha$ is the angle between the applied field direction and the horizontal direction. (a) A DW positioned before the cross in the left arm. A field $H$ at $\alpha=45^{\circ}$ moves the DW toward the cross. (b) A DW positioned in the right arm after moving through the cross. Schematic representation of (c) a headto-head DW and (d) a tail-to-tail DW. The color code indicates the in-plane magnetization direction, as shown by the color wheel.

i.e., parallel to some component of the applied field [see Fig. 2(a)]. Since both branches have the same width, the magnetization structure in the cross is stray-field-free. When the DW is to the left of the cross [Fig. 2(a)], there is a continuous magnetic flux from bottom to left and from right to top. The magnetization is along a $135^{\circ}$ direction in the core of the cross. When the DW has moved through the cross, the core magnetization is rotated by $90^{\circ}$ in the clockwise direction, thereby forming a stray-field-free state [Fig. 2(b)] again.

In Fig. 3, we present the second possible starting configuration. In this case, the magnetization of the vertical arm is in a high energetic state, as the magnetization is antiparallel to some component of the applied field. Nevertheless, the magnetization pattern of the cross is stray-field-free, and the core magnetization is directed in a $225^{\circ}$ direction, as shown in Fig. 3(a). As a result of this configuration, the magnetizations in the top, bottom, and right arms are in a higher energetic state. Therefore, the DW could travel horizontally or vertically (up or down), resulting in the three states shown in Figs. 3(b), 3(c), and 3(d). Only the movement in the horizontal path [Fig. 3(b)] yields a stray-field-free configuration (switching of the core magnetization by $90^{\circ}$, from $225^{\circ}$ to $315^{\circ}$ ). Any propagation of the DW into the up or down arms results in a magnetization structure with a $180^{\circ} \mathrm{DW}$ [Fig. 3(c)] or an antivortex state [Fig. 3(d)] in its center.

From an energetic point of view, the energy barrier to overcome in the final states in Figs. 3(c) and 3(d) is higher than in the sole horizontal depinning in Fig. 3(b). This higher energy barrier is due to the required energy to create an additional DW at the cross. From this simple picture, we intuitively expect a preferred DW motion in the horizontal direction toward the stray-field-free state in Fig. 3(b) instead of the more energetic states in Figs. 3(c) and 3(d). Despite those differing energy barriers for the different final states, if the applied field exhibits a large component along the
Cross with straight arms and critical magnetization structure

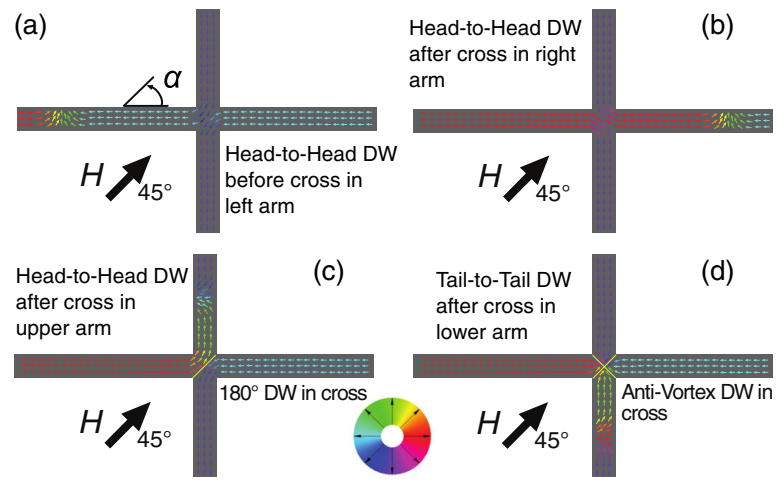

FIG. 3. Sketch of the DW configuration before and after moving through a cross with the possible resulting magnetization structures. $\alpha$ is the angle between the applied field direction and the horizontal direction. The applied field can remagnetize the horizontal arm or the vertical arm. (a) The DW is positioned before the cross in the left arm. A field $H$ inclined at $45^{\circ}$ moves the DW toward the cross. For $\alpha=45^{\circ}$, the DW can be positioned in any of the three arms after moving through the cross. (b) A DW placed in the right arm. (c) A DW placed in the upper (wrong) arm and (d) a DW positioned in the lower (wrong) arm. In (a) and (b), the cross is DW-free. In (c), a $180^{\circ} \mathrm{DW}$ and in (d) an antivortex DW is located in the center of the cross. Additionally, in (d), the head-to-head DW must transform into a tail-to-tail DW during the movement through the cross. The in-plane magnetization direction is indicated by the color code visible in the color wheel.

vertical direction, the DW is likely to move vertically, resulting in a failure of the operation of the sensor device. Therefore, it is desirable to constrain the DW propagation to angles of around $\alpha=0^{\circ}$ and $180^{\circ}$, respectively.

However, in a simple closed-loop multiturn counter, the DWs are moved by a rotating magnetic field. We consider, in this case, a clockwise rotation of the field. The motion in the horizontal nanowire starts depending on the natural pinning strength for a head-to-head DW in the wires at field directions of around $\alpha=70^{\circ}-80^{\circ}$ because the horizontal component of the applied field is sufficient to depin. Under these conditions, the DW would not reliably travel from the left arm to the right arm as desired, but instead into one of the vertical arms, as depicted in Figs. 3(c) and 3(d), resulting in a counting error of the sensor. To prevent this failure, we introduce an innovative alternative syphonlike geometry (Fig. 4).

This syphon has the aim of pinning the DW in its arms until the field angle is favorable for a propagation only in the horizontal direction. For example, in Fig. 4(a), with $\alpha=45^{\circ}$ and $\theta=45^{\circ}$ (syphon-arm angle), $\beta$, the angle that is perpendicular to the syphon arm, is $0^{\circ}$; thus, the applied field is perpendicular to the syphon arm, and the DW is pinned in the arm. In Fig. 4(b), with the applied field angle $\alpha=30^{\circ}$, the angle $\beta=15^{\circ}$; thus, there exists a component of the field along the syphon arm, and the DW can depin. At field angles lower than $\alpha=45^{\circ}$, a larger torque is 
Cross with $45^{\circ}$-syphon and critical magnetization structure

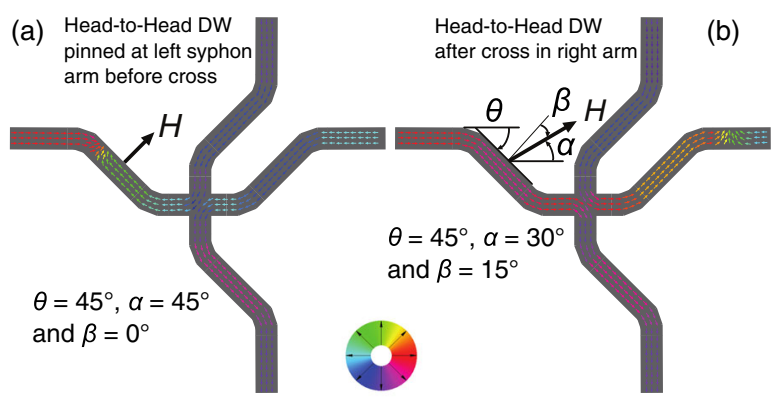

FIG. 4. Sketch of the DW motion through a cross combined with a $45^{\circ}$ syphon. $\theta$ is the angle of the syphon arm with respect to the horizontal direction, $\alpha$ is the field angle with respect to the horizontal direction, and $\beta$ is the angle between the perpendicular direction with the wire containing the DW and the applied field direction. (a) A DW is pinned at the beginning of the syphon in the left arm for $H$ inclined at $45^{\circ}$, resulting in no longitudinal component to move the DW into the syphon $\left(\beta=0^{\circ}\right)$. (b) The DW moved through the cross with $H$ inclined at $30^{\circ}\left(\beta=15^{\circ}\right)$.

provided in the horizontal direction than in the vertical, thus favoring horizontal propagation through the cross, as depicted in Fig. 4(b).

Thus far, this conceptual discussion compares quasistatic states. The complexity of the DW motion under an applied external field can also yield other transitions. Therefore, extensive micromagnetic simulations need to be performed to study the influence of the syphon geometry on the DW motion through the cross. The latter is then the key step to finding parameters that minimize failure and optimize reliability.

The entire previous discussion is based on an analysis of a head-to-head DW with $\alpha$ being between $90^{\circ}$ and $0^{\circ}$ or $0^{\circ}$ and $270^{\circ}$. By analogy, this analysis is equally applicable to a tail-to-tail DW with $\alpha$ being between $90^{\circ}$ and $180^{\circ}$ or $180^{\circ}$ and $270^{\circ}$.

\section{MICROMAGNETIC SIMULATIONS}

All of the simulations are performed with the software MUMAX3 [21] on graphics processing units. The material parameters used for $\mathrm{Ni}_{81} \mathrm{Fe}_{19}$ are as follows: the saturation magnetization $M_{s}=860 \mathrm{kA} / \mathrm{m}$, the exchange constant $A=1.3 \times 10^{-11} \mathrm{~J} / \mathrm{m}$, no magnetocrystalline anisotropy, and a Gilbert damping of 0.01 . The thickness of the material is $30 \mathrm{~nm}$, the width of the stripe is $300 \mathrm{~nm}$, and the cell size is kept below $5 \times 5 \times 15 \mathrm{~nm}^{3}$. The various relevant parts of the structure (the cross and the syphon) are separately investigated. A separate analysis is possible since the pinning potential in the syphon due to the corners is sufficiently strong to mask any attraction from the center of the cross. The two elements are then effectively decoupled and can be investigated individually in detail. While we used a transverse DW as a simple representation of a DW in the previous section, the cross section, as well as material used in the experiments and the micromagnetic simulations, will favor a vortex DW. All simulations are thus started with a vortex DW that corresponds to the experimentally expected wall spin structure.

\section{A. Crossing of two magnetic stripes}

Thus far, the poorly controlled nature of DW propagation through the cross without a syphon has been an effect that limits the use of $n$-CL structures for sensors. Despite some studies on the propagation of the DW in split paths [19,22-24], DW dynamics propagating through the center of the cross is not yet fully understood, particularly its behavior about the reversal of the vertical arm of the cross.

A systematic study of DW propagation is performed by changing both the applied field strength and the orientation. An initial configuration is obtained by placing a tail-to-tail vortex DW on the left arm and letting the system relax under no external applied field. A field with a chosen direction and magnitude is then applied, and the system is left to relax. Different testing conditions are then probed to determine whether the desired behavior is obtained, namely, the motion from left to right through the cross without the reversal of a vertical arm.

The orange circles represent the pinning of the DW in the center [Fig. 5(b) and Video 1], the red diamonds the reversal of the vertical arm [Fig. 5(c) and Video 3], and the blue diamonds the successful propagation through the cross [Figs. 2, 3(b), and Video 2]. We notice that, since the domain wall is tail to tail, the angular range for $\alpha$ is between $90^{\circ}$ and $270^{\circ}$ (centered around $180^{\circ}$ ), enabling the wall to propagate from left to right.

The simulations are performed for the angle range between $130^{\circ}$ and $230^{\circ}$, which is sufficient to observe

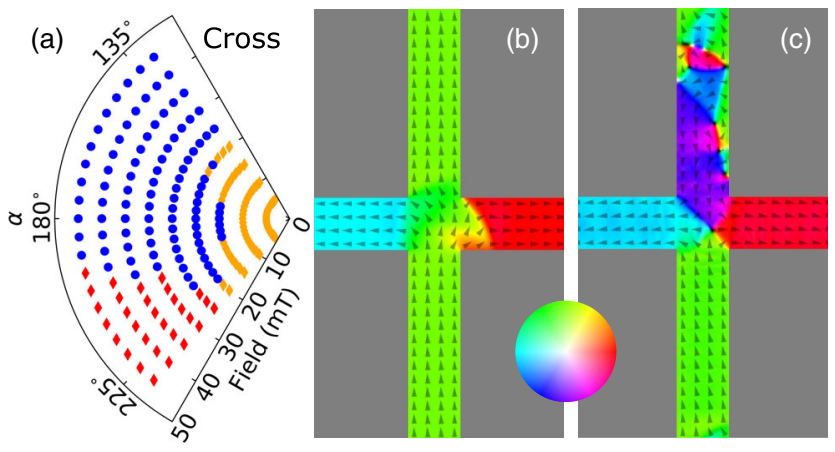

FIG. 5. (a) Polar plot of the angular dependence of a tail-to-tail DW propagation, with field orientation and magnitude represented as radius and angle, respectively. The blue circles indicate successful propagation through the cross; the red diamonds represent a reversal of the vertical arm, and the orange the pinning at the cross. (b) Spin structure of a DW pinned at a cross. (c) Snapshot of magnetization dynamics showing the splitting of the DW generating the reversal of the vertical arm in addition to the horizontal arm and thus representing a failure event. The inplane magnetization direction is indicated by the color code visible in the color wheel. 


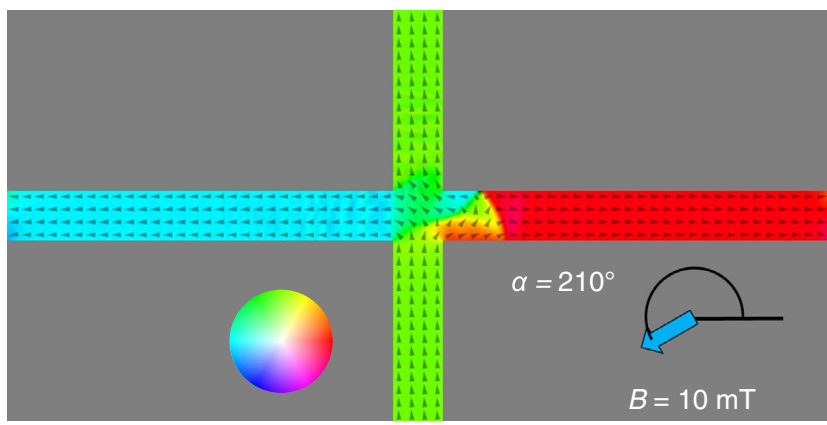

VIDEO 1. Snapshot of the magnetization with the DW pinned around in the center. Snapshot of the magnetization with the DW is pinned in the center of the cross-shaped element under an applied field with $\alpha=210^{\circ}$ and strength $=10 \mathrm{mT}$. The in-plane magnetization direction is indicated by the color code visible in the color wheel.

all the interesting switching behavior. The field amplitude is also kept below $45 \mathrm{mT}$, which is the nucleation-field value of a wire in our system extracted from the StonerWohlfarth model [25]. Indeed, if the field reaches higher values, DWs can nucleate in the closed system, destroying the two studied spin structures. The applied field can be rotated clockwise or counterclockwise in the device. However, from symmetry arguments, it is sufficient to scan one direction, so we scan the plot starting from $240^{\circ}$ toward $110^{\circ}$ for different magnitudes of the field. At the first data points $\left(230^{\circ}\right)$, only failure events are encountered, i.e., pinning below $20 \mathrm{mT}$ and vertical-arm reversal above. The first propagation is reached for $20 \mathrm{mT}$ at $220^{\circ}$.

Regarding field strengths, the first propagation occurs at $15 \mathrm{mT}$ for a range of $10^{\circ}$ centered around $180^{\circ}$, indicating that the lowest depinning field is between 10 and $15 \mathrm{mT}$ (not observable due to our field step size). We observe an increase of the angular range of propagation as the field strength increases. This tendency continues until $25 \mathrm{mT}$, where the first reversal of the vertical arm occurs. The depinning dependence appears to not be symmetrical around the $x$ axis due to the smaller applied torque to

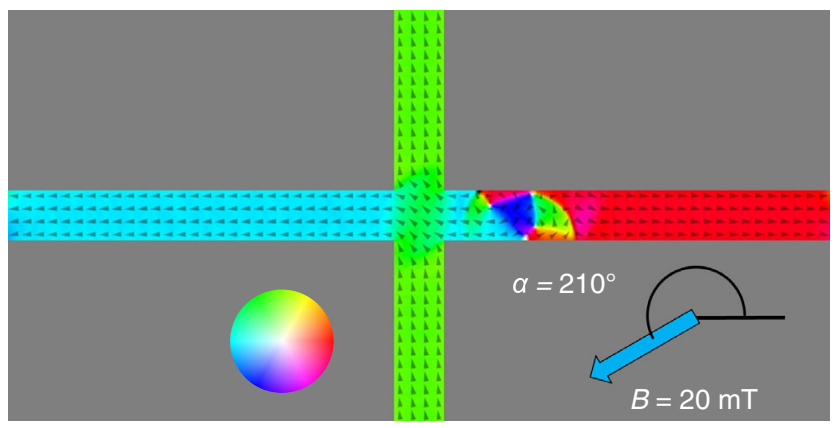

VIDEO 2. Snapshot of the magnetization dynamics showing the DW propagating through the center of the cross-shaped element under an applied field with $\alpha=210^{\circ}$ and strength $=$ $20 \mathrm{mT}$. The in-plane magnetization direction is indicated by the color code visible in the color wheel.

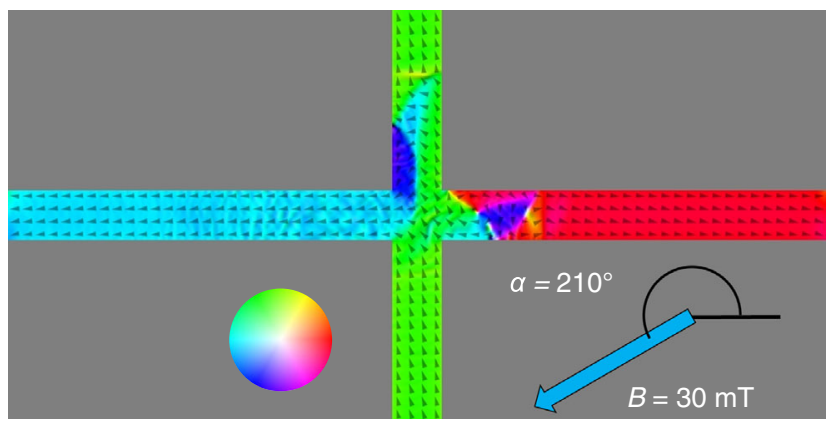

VIDEO 3. Snapshot of the magnetization dynamics showing the DW reversing the vertical arm under an applied field with $\alpha=210^{\circ}$ and strength $=30 \mathrm{mT}$. The in-plane magnetization direction is indicated by the color code visible in the color wheel.

the DW at lower angles while it is pinned in the center of the cross. At the entrance of the cross, the abrupt change in magnetostatics constitutes a potential well for the DW. The DW in the wire is confined to a pseudo-one-dimensional structure and keeps a well-defined vortex wall internal structure with two edge defects. At the cross, however, due to the lack of vertical confinement, one of the two edge defects annihilates and renucleates on the opposite side of the horizontal arm, while the other topological defect is spread across the vertical arm, effectively reducing the stray field [see Fig. 5(b)].

The fact that the half edge defect present in the stripe is always the one with the lower stray field thus supports our interpretation. Above $25 \mathrm{mT}$, the angular range decreases due to the large vertical component of the applied field, which promotes the propagation along the vertical arm. At $45 \mathrm{mT}$, an angular range of $10^{\circ}$ for the propagation is still present in the bottom quadrant. No reversal of the vertical arm is observed in the top quadrant because the vertical component of the applied field is parallel to the magnetization. Concerning the vertical-arm reversal, the variety of spin configurations [26,27] that the DW can acquire at high fields while interacting with the center of the cross makes the dynamics complex.

The average magnetization and the energy-densityterm evolution across time is shown in Fig. 6 for the three characteristic events described previously. As expected, the average $x$ component is larger for the pinning case than for the two others due to the incomplete reversal of the horizontal arm. Similarly, a strong change of the average $y$ component is observable in the vertical-reversal case. Considering the energy-density terms, the total energy density is mainly driven by the Zeeman energy density of the system. In the three cases, an increase in the Zeeman energy density first occurs due to the ramp-up of the applied field that follows a functional dependence of the form $B=\left\{B_{\text {ext }}\left[1-\exp \left(-t / 5 e^{-10}\right)\right] \cos (\alpha)\right.$, $\left.B_{\text {ext }}\left[1-\exp \left(-t / 5 e^{-10}\right)\right] \sin (\alpha), 0\right\}$. The following decrease is due to the DW movement switching the magnetization to a lower energy state. The magnitude of the Zeeman energy 

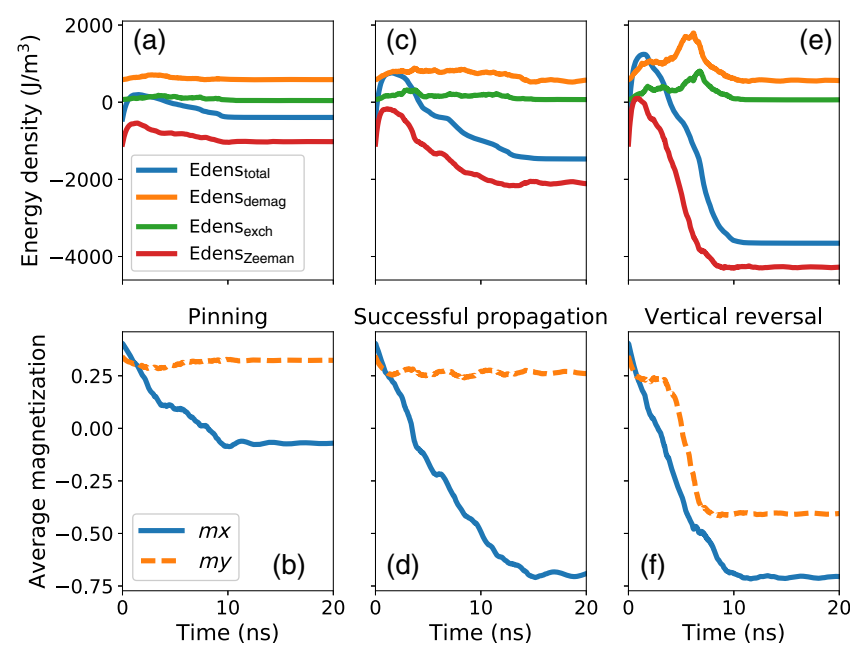

FIG. 6. Time evolution of (a) the energy-density terms and (b) $x$ and $y$ components of the magnetization in the case of the DW being pinned at the cross (field, $\alpha=210^{\circ}$; strength, $10 \mathrm{mT}$ ). Time evolution of (c) the energy-density terms and (d) the $x$ and $y$ components of the magnetization in the case of a DW propagating through the cross (field, $\alpha=210^{\circ}$; strength, $20 \mathrm{mT}$ ). Time evolution of (e) the energy-density terms and (f) the $x$ and $y$ components of the magnetization in the case of a DW inducing vertical-arm reversal (field, $\alpha=210^{\circ}$; strength, $30 \mathrm{mT}$ ).

density at equilibrium is then directly proportional to the amount of magnetic material switched in the direction of the applied field. For the pinned case, half of the horizontal wire is switched, for the successful propagation, the whole horizontal wire is switched, and, for the vertical reversal, the entire system is switched.

Owing to the rotating field used in the actual sensordevice concept, if the vertical arm has a magnetization antiparallel to the applied field, the reversal of the vertical arm is likely to occur, resulting in a failure event. For example, if a tail-to-tail DW is found to the left of the cross when a field of $40 \mathrm{mT}$ is applied along $200^{\circ}$, according to Fig. 5(a), its propagation will lead to a reversal of the vertical arm. To overcome this issue, we next introduce additionally the syphon element, which will constrain the propagation through the cross at applied field angles close to $180^{\circ}$.

\section{B. Syphon element}

The syphon structure comprises two horizontal wire segments connected by a tilted wire at an angle $\theta$ from the horizontal direction (Fig. 4). The goal is to limit the movement of the DW to field directions that lead to successful horizontal DW propagation without vertical DW propagation, as represented by the blue area in Fig. 5(a). The syphon arms are placed on each side of the cross to allow for the use of the device with a clockwise and counterclockwise rotating field.

In simulations, a DW is initialized at the top-left horizontal arm of the syphon and propagated toward the

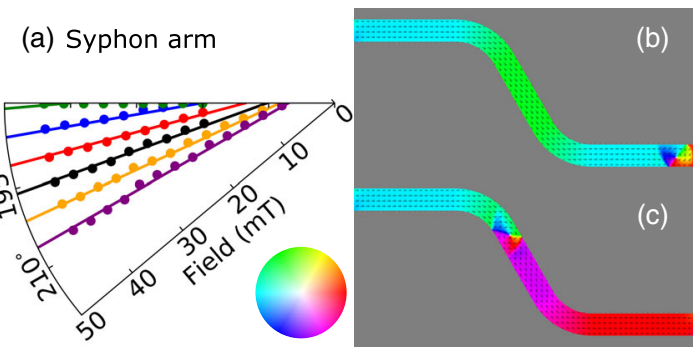

FIG. 7. (a) Polar plot depicting the limit between pinning and propagation at the syphon arms. The circles are simulation results for the syphon angle $\theta=85^{\circ}, 80^{\circ}, 75^{\circ}, 70^{\circ}, 65^{\circ}$, and $60^{\circ}$, respectively, in green, blue, red, black, orange, and purple. The lines are the fitting curves of the simulated data based on Eq. (1). Taking the results for $\theta=60^{\circ}$ (the purple line), the DW pinning occurs for field angles larger than $205^{\circ}$, the propagation is allowed for field angles between $180^{\circ}$ and $205^{\circ}$ and field strengths larger than $7 \mathrm{mT}$. (b) Successful propagation of the DW through the syphon. (c) Pinning at the center of the syphon. The in-plane magnetization direction is indicated by the color code visible in the color wheel.

right. The two final configurations are the desired propagation shown in Fig. 7(b) or the pinning of the DW in the center of the arm [Fig. 7(c)]. Different syphon angles $[\theta$ in Fig. 4(b)] are simulated. The results can be seen on the polar plot in Fig. 7(a). In Fig. 7(a), we present only the limit between pinning and propagation. The circles are the simulation results, while the lines are the fitting of the results for an angle of the syphon arm [Eq. (1)] with $\theta$ varying from $85^{\circ}$ to $60^{\circ}$ in steps of $5^{\circ}$ (the left syphon arm).

The results for a syphon with $\theta=60^{\circ}$, represented in purple, show that the domain wall will propagate through the syphon for field values between 7 and $45 \mathrm{mT}$ and angle values between $180^{\circ}$ and $205^{\circ}$ (the purple line). However, the domain wall will be pinned for applied fields at any angle higher than the limit marked by the solid line. The other syphon angles exhibit similar behavior, but with different limits.

We study the left arm of the syphon since all results can be applied to the right syphon by reflection symmetry along the vertical axis. Similarly, because of the symmetry of the system, if the angle of the applied field is smaller than $\alpha=180^{\circ}$, the DW will propagate through the first syphon arm and the cross, but not across the second syphon arm. Thus, we are then interested only in the angles where $\alpha$ is between $180^{\circ}$ and $220^{\circ}$. From the results, we find that the angular range increases with the magnitude of the applied field and the reduction of the syphon angle $(\theta)$.

The propagation of the DW through the syphon element is described by a model of a DW in a straight wire under the application of a field with axial and transverse components $[28,29]$. In a one-dimensional model [30,31], the torque on the DW from the applied field can be approximated as $\left.\mu_{0} H_{\text {ext }} M_{s} \sin \{[(3 \pi) / 2]-\theta-\alpha]\right\}$, and it is maximal when the applied field is parallel and opposite to the 
magnetization in the stripe. From the latter, we extract the depinning field in the arm:

$$
H_{\mathrm{ext}}=\frac{H_{p}}{\left|\sin \left(\frac{3 \pi}{2}-\theta-\alpha\right)\right|}
$$

with $H_{p}$ being the pinning field in the structure due to the corner geometry. For $H_{p}=3.5 \mathrm{mT}$, we notice the excellent agreement with the model. The syphon arm can thus prevent the DW from propagating for field directions outside the desired angular range. In a real device, the edge roughness might affect the propagation field in the syphon element. The result is likely to be a larger required longitudinal component of the field along the syphon arm to allow for the depinning of the DW. Although we have not investigated the effect of edge roughness, in our opinion, the behavior at the cross will not be affected, and that of the propagation fields through the syphon might increase a little, but the angular dependence will remain.

\section{FULL DEVICE GEOMETRY}

Finally, we now combine both syphon and cross elements for the creation of a reliable device geometry with the desired domain-wall propagation properties. The creation of the angular dependence of a complete device is simply accomplished by merging the angular dependences of the two elements. This merger is justified since the interaction between the elements is small due to the physical distance separating them. The results are shown in Fig. 8, where the following scheme is chosen to represent the results: if the DW propagates across both elements as desired, field direction and strength are represented by a green diamond. If a vertical reversal can still occur then, the diamonds are red. The black diamonds represent the configurations where
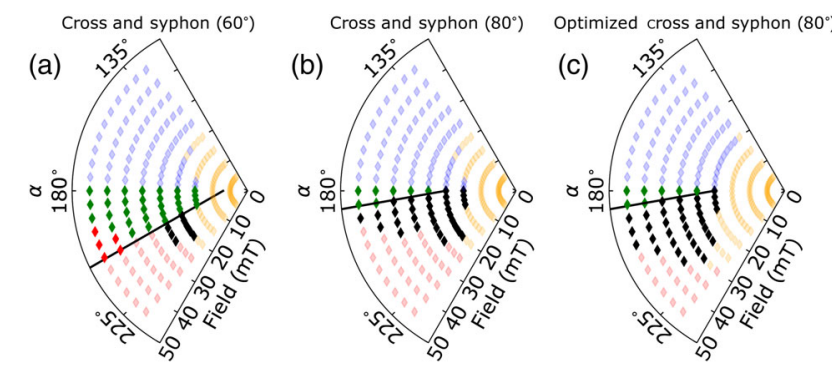

FIG. 8. (a) Polar plot of the combination of a cross with a syphon arm of angle $60^{\circ}$ (device no. 1). Orange represents pinning of the domain wall due to a field strength that is too low. Green represents the propagation through the full structure; the red circles are the reversal of the vertical arm, and the black region is where the device could potentially still be working if propagation through the syphon is facilitated. We refer to this region as a buffer zone. (b) A similar plot for a device with syphon angle $80^{\circ}$ (device no. 2 ). (c) A similar plot for an optimized device with a cross center of $210 \mathrm{~nm}$ and syphon angle $80^{\circ}$ (device 3 ). The black line is the fitting limit expressed previously for the chosen syphon arm. the DW would be pinned in the syphon arm but, if released in the cross, would still successfully propagate. We call this the buffer zone. All of the irrelevant points of the crossrelated effects are now colored with less intensity (in other words, faded). A device made of a certain cross and syphon is expected to function properly if no red diamonds (vertical reversal) are seen and if the buffer zone is large enough to account for stochastic events due to thermal activation and irregularities, which would broaden the micromagnetically computed working range boundaries.

The merging of a cross and a syphon arm with an angle $\theta=60^{\circ}$ yields the polar plot in Fig. 8(a) (device no. 1). We note that the field is rotating and that all nonfaded points need to be examined to assess whether a device is working. We notice that, in the allowed range of device no. 1, the application of a field larger than $25 \mathrm{mT}$ would lead to a reversal of the vertical arm since there is no buffer region above it. If we are to define an angle operating window (AOW) between the largest working angle $200^{\circ}$ (at $25 \mathrm{mT}$ ) and $180^{\circ}$, device no. 1 has an AOW of $20^{\circ}$. It is useful to define a field operating window (FOW) between the depinning field limit and the central-arm reversal limit. For device no. 1, the FOW is $10 \mathrm{mT}(15-25 \mathrm{mT})$. Finally, the robustness of the structure to processing irregularities and stochasticity can be identified by the smallest black angular range. For device no. 1, the robustness is on the order of $15^{\circ}$ at $25 \mathrm{mT}$. We believe that for a device, the best parameters are met for a large FOW, a small AOW, and a wide buffer zone or robustness to irregularities due to device fabrication. A summary of the values for those parameters are found in Table II.

The second plot [Fig. 8(b)] represents the characteristics of device no. 2. Device no. 2 is created by merging the cross to a syphon arm of $80^{\circ}$. This device has a passing field up to the theoretical nucleation field of the structure (no vertical-arm reversal). The FOW of device no. 2 is $25 \mathrm{mT}(20-45 \mathrm{mT})$, which represents a substantial improvement over device no. 1 . The AOW is also reduced to $10^{\circ}$. However, the buffer zone is only $5^{\circ}$ at $45 \mathrm{mT}$ and thus the operation is vulnerable to defects. The latter is due to the strong enlargement of the vertical-arm-reversal area as the magnitude of the field increases.

We now optimize the cross with the best syphon angle to enlarge the buffer region. The dimensions in the center of the cross are reduced from 300 to $210 \mathrm{~nm}$ (device no. 3). This reduction increases the nucleation field, and potentially also the propagation field. The results of the reduction are seen on

TABLE II. Summary of the three defining parameters of the $n$-CL device for the three presented architectures.

\begin{tabular}{lccc}
\hline \hline & No. 1 & No. 2 & No. 3 \\
\hline Angle operating window (deg) & 20 & 10 & 10 \\
Field operating window (mT) & 10 & 25 & 25 \\
Minimum buffer zone (deg) & 15 & 5 & 15 \\
\hline \hline
\end{tabular}


the faded points in Fig. 8(c). The depinning occurs at 5-mThigher values, but the angular range at $45 \mathrm{mT}$ is much larger. Because of the selection of the syphon angle, the increase in the depinning field has no impact on the performance of the device. The robustness of device no. 3 is then drastically improved to $15^{\circ}$ at $45 \mathrm{mT}$ compared to no. 2 , with no loss of FOW, thus providing the necessary reliability to the structure. Depending on the desired parameter range for the FOW, tailored combinations of a syphon and a cross structure allow for the creation of optimum conditions for maximum-reliability operation.

\section{CONCLUSION}

In this paper, we present an innovative alternative nonvolatile sensor concept based on magnetic DWs that will allow for counting up to millions of turns of an applied external rotating magnetic field. The concept requires the use of the cross-shaped intersections of nanowires forming intertwined loops. We explore the DW propagation in this cross-shaped geometry and obtain different states of the cross while interacting with a domain wall. We find that, with a simple cross, some configurations (where the applied field and the magnetization in the vertical arm are antiparallel) are generating the failure event for the sensor operation.

We develop a syphon structure comprising a tilted wire to overcome the problems leading to failure events. We simulate elements under fields of different strengths and angles to identify their operating conditions. The cross yields a complex DW behavior characterized by three physical mechanisms: the propagation through the cross, the pinning at the cross, and the reversal of a vertical arm. The syphon arm for different syphon angles is modeled via a hyperbolic dependence on the angle of the applied field. The syphon structure successfully allows for the pinning of the DW in angular ranges where the applied field configuration corresponding to the magnetization state in the cross would yield a failure if the domain wall were to propagate into the cross region at these field directions and field strengths.

Finally, the combination of the two elements allows for the identification of an angular operating window, a field operating window, and a robustness factor; the three of them allow for an easy identification of a reliable structure, which is essential for designing the geometries of reliable devices of this generation of multiturn sensor.

\section{ACKNOWLEDGMENTS}

The authors would like to acknowledge K. Litzius for helping with the simulations and the WALL project for financial support. The work and results reported in this publication were obtained with research funding from the European Community under the Seventh Framework Programme [Multi-ITN WALL Grant Agreement No. 60803] a European Research Council proof-of-concept grant [MultiRev ERC-2014-PoC (Grant No. 665672)], and the German Research Foundation (Grant No. SFB TRR173 Spin+X). L. L.-D. acknowledges the support of Project No. SA090U16 from Junta de Castilla y Leon.

[1] R. Spain, Domain tip propagation logic, IEEE Trans. Magn. 2, 347 (1966).

[2] R. Spain and M. Marino, Magnetic film domain-wall motion devices, IEEE Trans. Magn. 6, 451 (1970).

[3] Robert D. McMichael and Michael J. Donahue, Head to head domain wall structures in thin magnetic strips, IEEE Trans. Magn. 33, 4167 (1997).

[4] M. Kläui, Head-to-head domain walls in magnetic nanostructures, J. Phys. Condens. Matter 20, 313001 (2008).

[5] D. A. Allwood, Gang Xiong, M. D. Cooke, C. C. Faulkner, D. Atkinson, N. Vernier, and R. P. Cowburn, Submicrometer ferromagnetic NOT gate and shift register, Science 296, 2003 (2002).

[6] A. Allwood, G. Xiong, C. C. Faulkner, D. Atkinson, D. Petit, and R. P. Cowburn, Magnetic domain-wall logic, Science 309, 1688 (2005).

[7] J.-O. Klein, E. Belhaire, C. Chappert, R. Cowburn, Daniel Read, and D. Petit, Synthesis methodology for magnetic domain wall logic, Int. J. Electron. 95, 249 (2008).

[8] K. A. Omari and T. J. Hayward, Chirality-Based Vortex Domain-Wall Logic Gates, Phys. Rev. Applied 2, 044001 (2014).

[9] S. S. P. Parkin, Masamitsu Hayashi, and Luc Thomas, Magnetic domain-wall racetrack memory, Science 320, 190 (2008).

[10] Marco Donolato, Paolo Vavassori, Marco Gobbi, Maria Deryabina, Mikkel F. Hansen, Vitali Metlushko, Bojan Ilic, Matteo Cantoni, Daniela Petti, Stefano Brivio et al., On-chip manipulation of protein-coated magnetic beads via domainwall conduits, Adv. Mater. 22, 2706 (2010).

[11] Marco Diegel, Roland Mattheis, and Ernst Halder, 360 domain wall investigation for sensor applications, IEEE Trans. Magn. 40, 2655 (2004).

[12] Marco Diegel, Roland Mattheis, and Ernst Halder, Multiturn counter using movement and storage of 180 magnetic domain walls, Sens. Lett. 5, 118 (2007).

[13] Marco Diegel, Sacha Glathe, Roland Mattheis, Manfred Scherzinger, and Ernst Halder, A new four bit magnetic domain wall based multiturn counter, IEEE Trans. Magn. 45, 3792 (2009).

[14] R. Mattheis, S. Glathe, M. Diegel, and U. Hübner, Concepts and steps for the realization of a new domain wall based giant magnetoresistance nanowire device: From the available $2^{4}$ multiturn counter to a $2^{12}$ turn counter, J. Appl. Phys. 111, 113920 (2012).

[15] Novotechnik RSM-2800 Multiturn and RMB-3200, http://www.novotechnik.de/produkte/winkelsensoren/ (11 May 2017).

[16] R. Mattheis, Magnetic revolution counter, European Patent No. EP 2191237 B1 (2014).

[17] M. Diegel and R. Mattheis, Magnetic sensor for absolute counting of revolutions or linear distances, German Patent No. DE 102013018680 A1 (7 May 2015). 
[18] B. Borie, A. Kehlberger, J. Wahrhusen, H. Grimm, and M. Kläui, Geometrical Dependence of Domain-Wall Propagation and Nucleation Fields in Magnetic-Domain-Wall Sensors, Phys. Rev. Applied 8, 024017 (2017).

[19] E. R. Lewis, D. Petit, A.-V. Jausovec, L. O'Brien, D. E. Read, H. T. Zeng, and R. P. Cowburn, Measuring Domain Wall Fidelity Lengths Using a Chirality Filter, Phys. Rev. Lett. 102, 057209 (2009).

[20] E. R. Lewis, D. Petit, L. O'Brien, A. Fernandez-Pacheco, J. Sampaio, A. V. Jausovec, H. T. Zeng, D. E. Read, and R. P. Cowburn, Fast domain wall motion in magnetic comb structures, Nat. Mater. 9, 980 (2010).

[21] Arne Vansteenkiste and Ben Van de Wiele, MUMAX: A new high-performance micromagnetic simulation tool, J. Magn. Magn. Mater. 323, 2585 (2011).

[22] Aakash Pushp, Timothy Phung, Charles Rettner, Brian P. Hughes, See-Hun Yang, Luc Thomas, and S. S. P. Parkin, Domain wall trajectory determined by its fractional topological edge defects, Nat. Phys. 9, 505 (2013).

[23] D. M. Burn, M. Chadha, S. K. Walton, and W. R. Branford, Dynamic interaction between domain walls and nanowire vertices, Phys. Rev. B 90, 144414 (2014).

[24] P. Sethi, C. Murapaka, S. Goolaup, Y. J. Chen, S. H. Leong, and W. S. Lew, Direct observation of deterministic domain wall trajectory in magnetic network structures, Sci. Rep. 6, 19027 (2016).

[25] E. C. Stoner and E. P. Wohlfarth, A mechanism of magnetic hysteresis in heterogeneous alloys, Phil. Trans. R. Soc. A 240, 599 (1948).

[26] Norman L. Schryer and Laurence R. Walker, The motion of 180 domain walls in uniform de magnetic fields, J. Appl. Phys. 45, 5406 (1974).

[27] M. Zeisberger and R. Mattheis, Magnetization reversal in magnetic nanostripes via Bloch wall formation, J. Phys. Condens. Matter 24, 024202 (2012).

[28] Matthew T. Bryan, Thomas Schrefl, Del Atkinson, and Dan A. Allwood, Magnetic domain wall propagation in nanowires under transverse magnetic fields, J. Appl. Phys. 103, 073906 (2008).

[29] S. Glathe, I. Berkov, T. Mikolajick, and R. Mattheis, Experimental study of domain wall motion in long nanostrips under the influence of a transverse field, Appl. Phys. Lett. 93, 162505 (2008).

[30] A. Thiaville, J. M. Garcı, and J Miltat, Domain wall dynamics in nanowires, J. Magn. Magn. Mater. 242, 1061 (2002).

[31] Yoshinobu Nakatani, André Thiaville, and Jacques Miltat, Head-to-head domain walls in soft nano-strips: A refined phase diagram, J. Magn. Magn. Mater. 290, 750 (2005). 